\title{
ANXIETY, DEPRESSION AND RELATED FACTORS IN FEMALE CANCER PATIENTS IN ONCOLOGY OUTPATIENT SETTINGS IN TAIWAN
}

\author{
${ }^{1}$ Yun-Hsiang Lee; ${ }^{2} J u i-C h u n$ Chan; ${ }^{3}$ In-Fun Li; ${ }^{4}$ Hui-Hsuan Shih \\ 1School of Nursing, College of Medicine, National Taiwan University, Taipei
2RN, Lecturer, Department of Nursing, Mackay Medicine College, New Taipei \\ ${ }^{3} \mathrm{PhD}, \mathrm{RN}$, Vice Director, Mackay Memorial Hospital and Assistant Professor, Department of Nursing, Mackay Medicine College, Taipei \\ 4MD, Division of Chest and Critical Care Medicine, Department of Internal Medicine, Mackey Memorial Hospital, Taipei.
}

\section{Introduction}

- An increasing number of patients with cancer are being shifted from inpatient care to outpatient care.

- However, psychosocial care needs tend to be neglected in oncology outpatient settings.

\section{Objective}

- To examine the prevalence and severity of anxiety and depression in female patients with cancer being treated in outpatient settings in Taiwan. (aim 1)

- To identify factors related to anxiety and depression. (aim 2)

\begin{tabular}{|l|}
\hline \multicolumn{1}{|c|}{ A cross-sectional study and self-management } \\
theory was used to select measured variables. \\
- Eligible patients were recruited from oncology \\
outpatient settings in a medical center in \\
Taiwan. \\
- Questionnaires \\
1) HADS (Anxiety \& Depression) \\
2) MOS SSS (Social Support) \\
3) Utilization of Social Resources \\
- Conducting the Cancer Resource Center \\
(yes/No) \\
- Attending social supportive group (yes/No) \\
4) Self-management ability \\
- Linear regression was conducted to identify \\
factors related to anxiety and depression.
\end{tabular}

Table 1 Demographic and Clinical Characteristics in Female Cancer Patients ( $N=116)$

\begin{tabular}{|c|c|c|c|c|}
\hline Variable & $\mathbf{n}$ & $\%$ & Mean (SD) & Range \\
\hline Age (years) & & & $49.1(8.1)$ & 28-65 \\
\hline Education level (year) & & & $12.4(3.4)$ & 3-18 \\
\hline \multicolumn{5}{|l|}{ Married } \\
\hline Yes & 85 & 73.3 & & \\
\hline No & 31 & 26.7 & & \\
\hline \multicolumn{5}{|l|}{ Employed } \\
\hline Yes & 53 & 45.7 & & \\
\hline \multirow{2}{*}{\multicolumn{5}{|c|}{ Religion }} \\
\hline & & & & \\
\hline Yes & 85 & 73.3 & & \\
\hline No & 31 & 26.7 & & \\
\hline \multicolumn{5}{|l|}{ Comorbidity } \\
\hline Yes & 35 & 30.2 & & \\
\hline No & 81 & 69.8 & & \\
\hline \multicolumn{5}{|l|}{ Cancer type } \\
\hline Breast & 80 & 69.0 & & \\
\hline Lung & 19 & 16.4 & & \\
\hline Other & 17 & 14.6 & & \\
\hline \multicolumn{5}{|l|}{ Cancer stage } \\
\hline I & 28 & 24.1 & & \\
\hline II & 32 & 27.6 & & \\
\hline III & 26 & 22.4 & & \\
\hline IV & 30 & 25.9 & & \\
\hline \multicolumn{5}{|l|}{ Present treatment } \\
\hline Chemotherapy & 48 & 41.4 & & \\
\hline Radiotherapy & 21 & 18.1 & & \\
\hline Targeted therapy & 29 & 25.0 & & \\
\hline Other & 18 & 15.5 & & \\
\hline Performance status (ECOG) & & & $0.5(0.5)$ & $0-2$ \\
\hline 0 & 55 & 47.4 & & \\
\hline 1 & 60 & 51.7 & & \\
\hline 2 & 1 & 0.9 & & \\
\hline
\end{tabular}

\section{Results}

- For aim 1 (see Table 2)

1) Overall, $17.2 \%$ and $21.6 \%$ of the patients were at risk of anxiety and depression.

2) The average levels of anxiety and depression were $4.2(S D=4.1)$ and $4.1(S D=4.0)$, respectively.

- For aim 2 (See Table 3)

1) The patients who had comorbidity, poorer physical function, who did not attend a social support group, had less social support, lower self-management ability, reported higher levels of anxiety; the total variance explained by these results was $41.9 \%$.

2) The patients who had poorer physical function, who did not attend a social support group, less social support, and lower self-management ability reported higher levels of depression; in this case, the total variance explained was $47.6 \%$.

Table 2 The Prevalence and Levels (Severity) of Anxiety and Depression ( $N=116)$

\begin{tabular}{|lcr|}
\hline Variable & $\mathrm{n}(\%)$ & Mean (SD) \\
Anxiety (0-21) & & $4.2(4.1)$ \\
Non-cases (0-7) & $96(82.8)$ & $2.7(2.6)$ \\
Borderline cases (8-10) & $10(8.6)$ & $8.6(0.7)$ \\
Clinical cases (11-21) & $10(8.6)$ & $13.3(2.2)$ \\
Depression (0-21) & & $4.1(4.0)$ \\
Non-cases (0-7) & $91(78.4)$ & $2.3(2.6)$ \\
Borderline cases (8-10) & $19(16.4)$ & $9.3(1.0)$ \\
Clinical cases (11-21) & $6(5.2)$ & $14.3(2.0)$ \\
\hline
\end{tabular}

Table 3 Factors associated with anxiety and depression in female cancer patients $(N=116)$

\begin{tabular}{llll} 
& $\beta$ & $t$ & Sig. \\
Anxiety & & & \\
$\quad$ Comorbidity (yes vs. no) & 0.162 & 2.218 & 0.036 \\
Physical function (ECOG) & 0.249 & 3.055 & 0.003 \\
Attending social support group (yes vs. no) & -0.160 & -2.141 & 0.035 \\
Social support & -0.332 & -4.238 & $<0.001$ \\
Self-management ability & -0.248 & -3.006 & 0.003 \\
\hline$R^{2}=0.419$ & $\mathrm{~F}=15.117$ & & $<0.001$ \\
\hline Depression & & & \\
$\quad$ Physical function (ECOG) & 0.177 & 2.321 & 0.022 \\
Attending social support group (yes vs. no) & -0.148 & -2.106 & 0.038 \\
Social support & -0.421 & -5.781 & $<0.001$ \\
Self-management ability & -0.329 & -4.246 & $<0.001$ \\
\hline$R^{2}=0.476$ & $\mathrm{~F}=24.090$ & & $<0.001$ \\
\hline
\end{tabular}

\begin{tabular}{|c|}
\hline GOHGLSE'On \\
\hline $\begin{array}{l}\text { Approximately one-fifth of the female patients } \\
\text { were at risk of anxiety and depression. } \\
\text { - The factors related to anxiety and depression } \\
\text { that were identified in this study should be } \\
\text { addressed. }\end{array}$ \\
\hline
\end{tabular}

